\title{
Pronóstico de la demanda de turismo espacial suborbital en México: resultados de un estudio a través de Internet
}

\author{
María Angélica Piñón-González* \\ Universidad del Golfo de México (México) \\ José María Filgueiras-Nodar* \\ Universidad del Mar (México)
}

\begin{abstract}
Resumen: Este artículo muestra los resultados de una investigación llevada a cabo en Internet entre febrero y junio de 2016. Se comienza presentando el turismo espacial suborbital (TS) y justificando el interés de su estudio para el caso de México. Posteriormente, se presentan aquellos estudios de mercado del turismo espacial que se consideran antecedentes de la presente investigación. Después de exponer la metodología seguida, se muestra el pronóstico a diez años vista de la demanda de TS en México, a través de tres escenarios: base, en el cual viajarían al espacio 422 pasajeros, generando un total de 1,688 millones de pesos mexicanos (MDP); ampliado, en el que volarían 1,334 pasajeros, generando ingresos por 5,336 MDP.; y restringido, con 95 pasajeros e ingresos de 380 MDP. al final del período temporal elegido. El texto cierra con unas reflexiones finales, en las que se discute la utilidad de los datos obtenidos.
\end{abstract}

Palabras Clave: Turismo espacial; Investigación de mercados; Vuelos suborbitales; Virgin Galactic; XCOR Aerospace.

Forecast of the demand for suborbital space tourism in Mexico: results of a study through the Internet

Abstract: This article presents the results of research carried out on the Internet between February and June 2016. It begins by presenting suborbital space tourism (ST) and justifying the interest of its study for Mexico. Subsequently, we present the relevant market studies of space tourism. After explaining the methodology followed, the 10-year forecast of ST demand in Mexico is presented over three scenarios: the base, where 422 passengers would travel to space, generating a total of 84.4 million dollars (MD); the expanded version where 1,334 passengers would fly, generating revenues of $266.8 \mathrm{MD}$.; and the restricted scenario, withonly 95 passengers and revenues of $19 \mathrm{MD}$. at the end of the chosen time period. The text closes with some final reflections, where the usefulness of the data obtained is discussed.

Keywords: Space tourism; Market research; Suborbital flights; Virgin Galactic; XCOR Aerospace.

\section{Introducción}

El turismo espacial (TE) es una modalidad turística que nace en el año 2001, cuando el financiero estadounidense Dennis Tito realiza una estancia de siete días en la Estación Espacial Internacional (EEI), sufragada por sus propios medios (Randolph, 2003). Esta primera experiencia de TE, así como las seis que le siguieron, están referidas a la modalidad denominada turismo orbital (TO) (Anderson

Licenciada en Administración Turística por la Universidad del Mar (campus Huatulco). Estudiante de la Maestría en Mercadotecnia Turística en la misma institución. Profesora de Agencias de Viajes en la Universidad del Golfo de México (campus Huatulco); E-mail: mariapg@chahue.

** Profesor-Investigador de Tiempo Completo de Mercadotecnia Turística en la Universidad del Mar (campus Huatulco), miembro nivel 1 del Sistema Nacional de Investigadores; E-mail: jofilg@huatulco.umar.mx 
y Piven, 2005; Seedhouse, 2008), en la cual los turistas permanecen en una estructura capaz de dar vueltas alrededor del planeta.

La presente investigación se centra en una modalidad diferente, denominada turismo espacial suborbital (TS). Ghoshal (2007: 58) define el vuelo espacial suborbital como "una misión que permite al Vehículo de Lanzamiento Reutilizable volar fuera de la atmósfera, pero no permite alcanzar la velocidad necesaria para sostener una órbita continua de la tierra". Leonard (citado en Umashankar, 2007), por su parte, explica que los vuelos suborbitales se elevan verticalmente hasta el límite del espacio, al triple de la velocidad del sonido. Entonces, reducen su velocidad antes de llegar a su altura máxima. Los pasajeros pueden experimentar unos minutos de ingravidez entre el punto en que la nave comienza a reducir su velocidad y el punto en que la nave frena su marcha para reentrar en la atmosfera terrestre.

Una de las razones por los cuales esta investigación se centró en el TS se debe a los costos del producto turístico: en el caso del TO los precios hasta ahora han oscilado entre los 20 y los 35 millones de dólares, y los próximos viajes se considera que iniciarán a un costo de al menos 50 millones (Filgueiras, 2015). En cambio, los precios que se están manejando para el TS oscilan entre 200,000 y 250,000 USD (Filgueiras, 2016), lo cual, aunque sigue siendo muy costoso, amplía en gran medida el volumen de población que podría acceder a este tipo de turismo.

Aunque en la historia de la exploración espacial, los vuelos suborbitales se produjeron antes que los orbitales, en el mundo del turismo la situación se invirtió y, mientras que desde el 2001 ha sido posible realizar TO, el TS ha tenido que esperar, sin que de hecho haya llegado a realizarse todavía ningún vuelo comercial de estas características. No obstante, diversas empresas se encuentran trabajando con el objetivo de ofrecer cuanto antes sus vuelos suborbitales de carácter turístico. Entre las mismas se hallan EXOS Aerospace, Airbus Defence and Space o Blue Origin (Seedhouse, 2014), además de las dos que se consideran más cerca de lograr ese objetivo: XCOR Aerospace (Seedhouse, 2016) y Virgin Galactic (Seedhouse, 2015).

Esta empresa, propiedad del británico Richard Branson, utiliza la tecnología de cohetes reutilizables que ganó el reconocido Premio X Ansari en 2004 (Sadeh y Sadeh, 2011), y desde entonces ha comunicado en diversas ocasiones el inicio inminente de sus operaciones comerciales, que anunció para el 2009, 2010, 2012 y 2015, generando siempre grandes expectativas. No obstante, estas previsiones se mostraron demasiado optimistas (Filgueiras, 2015), y Virgin ha tenido que retractarse una y otra vez de sus anuncios. El 31 de octubre de 2014, además, se produjo un accidente que destruyó su nave Spaceship Two, causando la muerte de uno de los pilotos (Excelsior, 2014), lo cual obligó a la empresa a retrasar una vez más el comienzo de sus vuelos turísticos. A pesar de ello, en el momento en que se produjo el accidente, Virgin tenía ya más de 700 clientes de todo el mundo (Excelsior, 2014), incluyendo a tres mexicanos, que habían pagado el depósito de 20,000 USD para reservar una plaza en sus vuelos espaciales o directamente la totalidad del precio $^{1}$.

¿Cuáles son las posibilidades comerciales del TS de cara al futuro? ¿Existe realmente un mercado capaz de sostener el modelo de negocio propuesto por empresas como Virgin Galactic o XCOR Aerospace? Para responder a estas cuestiones, se necesitan estudios de mercado capaces de estimar con la mayor precisión que sea posible ${ }^{2}$ las cifras de demanda potencial, así como determinados aspectos clave del comportamiento del consumidor. Con respecto a la primera dimensión, que será la tratada en este artículo, debe decirse que ya existen numerosos estudios de tales características (a los cuales se hará referencia en el siguiente epígrafe). Tales estudios proporcionan conclusiones francamente optimistas con respecto al volumen del mercado futuro del TS. Sin embargo, se trata de estudios llevados a cabo en Japón, Canadá y, sobre todo, los Estados Unidos, pero nunca en algún país latinoamericano, incluyendo a México.

El interés de realizar un estudio como el presente en México puede justificarse recordando que, según la Asociación Mexicana de Agencias de Investigación de Mercado, el 7.2\% de la población del país pertenece al nivel socioeconómico A/B, el de mayor "capacidad social y económica" (López, 2009: 4). Por su parte, Wealthinsight (citada en La Jornada, 2013), firma dedicada a monitorear a los individuos con mayor riqueza del globo, afirmó en 2013 que en México existían más de 145,000 millonarios (definidos como personas cuyo patrimonio, sin incluir su residencia principal, supera el valor de 1 mdd.) y 2,540 multimillonarios $^{3}$. En 2014, según la misma fuente, el número de millonarios se incrementó en un $7 \%$ (Wealthinsight, 2014). Tales cifras son significativas como punto de partida, pues muestran la existencia de un segmento amplio de consumidores con la capacidad económica para acceder al TS en las condiciones de precio propuestas en la actualidad por las empresas oferentes.

No obstante, se precisan más investigaciones enfocadas en indicar cuántas de estas personas podrían estar realmente interesadas en comprar vuelos suborbitales. Proporcionar un pronóstico de tal demanda 
potencial es precisamente el objetivo de la presente investigación, que comenzará presentando un panorama de los principales estudios de mercado del TE realizados hasta el momento.

\section{Antecedentes}

Se han elaborado estudios sobre el mercado del TE desde la década de los '60 (el primer estudio conocido, realizado por Kraft Ehricke, se llevó a cabo en 1967). Asimismo, en los años '80 se desarrollaron numerosos estudios, como el de David Ashford en 1984, o el American Express en 1986. A continuación, se mencionarán los estudios que han sido tomados en cuenta para la presente investigación:

- Patrick Collins y colaboradores: este economista británico dirigió en 1993 el primer estudio de mercado que abordó en profundidad el tema del TE, trabajando para el Laboratorio Aeroespacial Nacional de Japón (Collins, Iwasaki, Kanayama y Ohnuki, 1994) y posteriormente el primer estudio de la demanda potencial de TE en Estados Unidos y Canadá (Collins, Stockmans y Maita, 1995), con el fin de comparar sus resultados con los obtenidos en Japón. El primer estudio se realizó a través de cuestionarios cubiertos personalmente, y el segundo mediante encuesta telefónica, utilizando en los dos casos muestreo probabilístico. Ambas investigaciones obtuvieron resultados similares, en el sentido de un elevado interés por el TE: una de sus conclusiones es que si los precios se pudieran reducir hasta un rango de entre 10,000 y 20,000 USD por persona, la demanda podría llegar a ser de un millón de pasajeros anuales.

- Futron \& Zogby: esta consultora llevó a cabo en 2002 (poco después del viaje de Dennis Tito a la EEI) una de las investigaciones más completas que se ha realizado sobre TE, tratando de responder objetivamente a cuestiones como el tamaño del mercado, el potencial de crecimiento y el perfil de los consumidores. Para ello, se entrevistó telefónicamente a muestra de 450 personas de elevados ingresos o patrimonio, con la intención de llegar a conclusiones generalizables. En este estudio se aplicó una estrategia en dos fases que ha sido ampliamente utilizada en investigaciones posteriores, incluida la presente, como es el mostrar en primer lugar los aspectos positivos del TE y en segundo lugar los negativos, comparando el interés suscitado tras las dos descripciones. En el caso del TS, por ejemplo, el porcentaje de personas que "definitivamente" realizarían vuelos suborbitales, desciende un 5\% entre ambas descripciones (Beard y Starzyk, 2002: 13). Futron \& Zogby trató de ponderar también las diferentes experiencias (positivas y negativas) vividas durante el vuelo espacial, así como de determinar las razones por las que la gente podría realizar TE, en cualquiera de sus dos modalidades, entre las cuales se destacan el hecho de ser pioneros y de poder ver la Tierra desde el espacio. El estudio, asimismo, pronostica la demanda para las dos modalidades de TE. En el caso del TS, se obtuvieron los resultados que muestra la Tabla 1. El estudio se cierra mencionando que los pronósticos serán mucho más precisos una vez el TS se ofrezca realmente en el mercado, dado que su demanda depende de factores que no se pueden conocer hasta que llegue ese momento, como puede ser el número de empresas que presten el servicio, el precio de los billetes o los requisitos de salud que se establezcan para realizar los vuelos.

Tabla 1: Pronóstico de la demanda de TS entre 2006 y 2021 según Futron \& Zogby, con precios en miles de dólares e ingresos en millones de dólares.

\begin{tabular}{|l|c|c|c|c|c|c|c|c|c|c|c|c|c|c|c|c|}
\hline & $\mathbf{2 0 0 6}$ & $\mathbf{2 0 0 7}$ & $\mathbf{2 0 0 8}$ & $\mathbf{2 0 0 9}$ & $\mathbf{2 0 1 0}$ & $\mathbf{2 0 1 1}$ & $\mathbf{2 0 1 2}$ & $\mathbf{2 0 1 3}$ & $\mathbf{2 0 1 4}$ & $\mathbf{2 0 1 5}$ & $\mathbf{2 0 1 6}$ & $\mathbf{2 0 1 7}$ & $\mathbf{2 0 1 8}$ & $\mathbf{2 0 1 9}$ & $\mathbf{2 0 2 0}$ & $\mathbf{2 0 2 1}$ \\
\hline Pax. & 356 & 455 & 591 & 769 & 999 & 1,298 & 1,685 & 2,186 & 2,830 & 3,656 & 4,711 & 6,048 & 7,770 & 9,916 & 12,545 & 15,712 \\
\hline Precio & 100 & 100 & 100 & 100 & 100 & 100 & 95 & 90 & 85 & 80 & 75 & 70 & 65 & 60 & 55 & 50 \\
\hline Ingresos & 36 & 46 & 59 & 77 & 100 & 130 & 160 & 197 & 241 & 293 & 353 & 423 & 505 & 595 & 690 & 786 \\
\hline
\end{tabular}

Fuente: Beard y Starzyk (2002: 52).

- SpaceWorks Engineering: el estudio elaborado en 2006 por esta consultora contó con la participación de seis expertos en el tema y 99 personas del público en general, siendo el primero que se desarrolló a través de Internet (en la web surveymonkey.com). Se trató de construir un modelo de la demanda de TO en hoteles orbitales y estaciones espaciales, teniendo en cuenta la incertidumbre que envuelve a un producto turístico tan novedoso. Entre otros aspectos, el estudio señala en sus 
previsiones de demanda que los clientes generalmente estarían más interesados en hacer TO si se contase con un hotel orbital, de modo que la existencia de tales facilidades incrementaría el tamaño del mercado (Ghoshal, 2007).

- The Adventurer's Studio: esta investigación, desarrollada por Webber y Reinfert (2006), para la consultora Spaceport Associates, también se llevó a cabo a través de Internet. A diferencia del estudio de Futron \& Zogby, éste no buscaba obtener conclusiones extrapolables, por lo cual asumió la existencia de un sesgo en el muestreo, al centrarse en los visitantes de la web incredibleadventures. com (es decir, personas interesadas en el turismo de aventura, quienes por otra parte parecerían constituir uno de los segmentos más interesantes para la futura industria del TE). Así, en el estudio participaron 998 personas (de las cuales: $91 \%$ hombres, $63 \%$ estadounidenses, $94 \%$ menores de 60 años, 14\% millonarios), casi todas con un historial de realización de actividades de riesgo, como el alpinismo. A esta muestra se le preguntó acerca diversos tópicos, como el diseño de las naves espaciales (las diferentes posibilidades de despegue y aterrizaje), el tiempo de entrenamiento requerido para un vuelo suborbital (59\% consideraron aceptable el dedicarle dos semanas), las actividades extravehiculares o "paseos espaciales" (que interesaron a un $88 \%$ del total) o el precio considerado "justo" para un vuelo suborbital (que se ubicó en un rango de 50,000 USD o menos, muy por debajo de los precios que se manejan).

- University of Petroleum and Energy Studies (UPES): este estudio, publicado en 2010, que elaboró el Centro para Estudios de la Aviación de la UPES (India), incluye un análisis del potencial de mercado de gran interés para la presente investigación, al aportar pronósticos sobre la demanda potencial y los posibles ingresos generados por el TE en dicho país asiático. En este estudio se aplicó un cuestionario a una muestra de 150 individuos, seleccionados de un universo de 84,000 ciudadanos indios con patrimonio superior a un millón de dólares (mdd.). Ya que sólo se pudieron recoger datos de un 10\% de tales individuos, se simuló el resto de respuestas, con base en la correlación de las mismas con aspectos como la edad o profesión de los participantes. Para establecer el mercado potencial de TS entre 2010 y 2025, se tomó como base el universo mencionado y se fue modificando de acuerdo con factores como el interés mostrado en las encuestas (especialmente después de escuchar los aspectos negativos del vuelo), las posibles reducciones de tal interés a lo largo del tiempo, debidas al hecho de que muchas personas consideran importante el ser "pioneros" o la aptitud física, compensando todo ello con el incremento en el número de personas con patrimonio superior a 1 mdd. en el período pronosticado. En el caso del TS, se estimó un precio inicial de 200,000 USD en el 2010, que se iría reduciendo hasta la mitad en el 2020. Los resultados muestran cómo, desde un inicio de 4,191 pasajeros (que generan unos ingresos de 629 mdd.) en el primer año considerado (2013), la demanda va creciendo paulatinamente hasta alcanzar en el 2025 un total de 49,749 pasajeros y unos ingresos de 4,975 mdd. con un crecimiento muy marcado entre los años 2020 y 2021, en los cuales la demanda casi se triplica (Jakhu, 2010).

- The Tauri Group: esta consultora realizó en 2012 un pronóstico de la demanda de vehículos suborbitales reutilizables (no limitado al TE). Para su pronóstico sobre el segmento de pasajeros en vuelos espaciales comerciales, Tauri (2012) diferenció dos grupos de población que podrían estar interesados en los vuelos suborbitales: individuos con un patrimonio superior a los 5 mdd. y entusiastas del espacio con un patrimonio inferior. Se desarrolló una encuesta que fue respondida por 226 miembros del primer grupo, tratando de descubrir su interés y disposición a comprar un vuelo. Para considerar a un individuo lo suficientemente interesado en realizar un vuelo suborbital, aquél habría tenido que responder que se hallaba extremadamente o muy interesado en realizarlo, que probablemente compraría uno de esos vuelos, que estaba dispuesto a pagar por ello 100,000 USD o más por el mismo, y que valoraba los vuelos espaciales por encima de otras opciones turísticas. Se hicieron también dos preguntas abiertas (“¿cuánto imagina gastar por persona en un viaje de una vez en la vida?” y “cuál ha sido su mayor gasto por persona en un viaje o experiencia?"), seleccionando a quienes respondieron más de 100,000 USD a la primera, y más de 50,000 a la segunda. El 5\% de los encuestados cumplió con los requisitos de estas últimas preguntas, mientras que otro $5 \%$ cumplió con las cuatro condiciones señaladas antes. Con estos datos, Tauri estimó el porcentaje de la población que estaría interesado en los vuelos suborbitales y además dispuesto a comprarlos, y después aplicó el resultado a los tres millones de individuos con un patrimonio superior a los 5 mdd. a nivel mundial, lo cual arrojó un estimado de alrededor de 8,000 personas. Para calcular la distribución temporal de los vuelos, se tomó en cuenta la edad promedio de los participantes (55 años) y se asumió que realizarían sus vuelos en los próximos 25 años (antes de cumplir 80): de este modo, un 40\% de los individuos realizarían su 
vuelo en los 10 años cubiertos por el pronóstico ${ }^{4}$. Con respecto a los entusiastas del espacio con menor nivel económico, los investigadores concluyeron que representarían un 5\% de la población del primer grupo. Usando los datos expuestos, se elaboró un escenario base para la demanda y dos alternativos, de mayor y menor crecimiento: el primero de éstos asumió la existencia de un mayor interés por el TS y disminuyó el nivel de gasto previo en una experiencia o viaje, mientras que en el de menor crecimiento supusieron un empeoramiento de la economía mundial. Los investigadores aunaron estas cifras a las estimadas para otros segmentos establecidos dentro del mercado de vuelos espaciales comerciales individuales: promociones, programas empresariales de incentivos, y entrenamiento de astronautas pertenecientes a agencias espaciales; la previsión de tal demanda se muestra en la Tabla 2. Se reconoce también la existencia de factores que aportan incertidumbre a sus cálculos: el inicio de los vuelos podría incrementar las compras; la mercadotecnia o los cambios en la percepción de la seguridad de los vuelos podrían modificar las previsiones. Lo mismo sucede con el precio, ya que la previsión se basa en los precios actuales, que podrían descender de manera abrupta. Tabla 2: Escenarios de previsión a 10 años de la demanda de vuelos espaciales
comerciales individuales expresada en pasajeros según The Tauri Group.

\begin{tabular}{|c|c|c|c|c|c|c|c|c|c|c|}
\hline & 1er. año & $2^{\circ}$ & $3^{\circ}$. & $4^{\circ}$ & $5^{\circ}$ & $6^{\circ}$ & $7^{\circ}$ & $8^{\circ}$ & $9^{\circ}$. & $10^{\circ}$ \\
\hline Base & 340 & 344 & 353 & 359 & 366 & 372 & 379 & 385 & 392 & 399 \\
\hline Crecimiento & 1,046 & 1,060 & 1,079 & 1,099 & 1,118 & 1,138 & 1,159 & 1,179 & 1,200 & 1,222 \\
\hline Limitado & 187 & 188 & 191 & 195 & 198 & 202 & 205 & 209 & 213 & 216 \\
\hline
\end{tabular}

Fuente: Tauri (2012: 36).

Después de haber expresado los principales antecedentes de esta investigación, se expondrá a continuación la metodología seguida en la misma.

\section{Metodología}

La presente es una investigación descriptiva (diagnóstica), no experimental y transeccional. Habiendo analizado los problemas generales del muestreo en Internet, así como las críticas y réplicas suscitados por los mismos (Burns y Bush, 2003; Jiménez y Puente, 2007; Zikmund y Babin, 2009; Estaella y Ardévol, 2011; Morales, 2011) se optó por utilizar el muestreo voluntario (de tipo no probabilístico), ampliamente utilizado en investigaciones tanto online como offline ${ }^{5}$. Desde luego, se reconoce que los resultados obtenidos en esta investigación no representan cifras estadísticamente válidas, sino estimaciones. Éstas se han tratado de establecer de la manera más verosímil que fue posible, y se ha optado por explicitar los sesgos y problemas existentes, de modo que estos aspectos puedan ser tenidos en cuenta por los lectores.

El método elegido para la recolección de los datos fue la encuesta, debido sobre todo a su flexibilidad (Alvira, 2011) y al grado de control que posibilita su estandarización (Díaz, 2005) ${ }^{6}$. Así, se desarrolló un cuestionario piloto, validado a través de su aplicación en el mes de enero de 2016 a 30 profesores del Instituto de Turismo de la Universidad del Mar (Huatulco, Oaxaca, México). Con la retroalimentación producto de sus comentarios, se construyó un nuevo cuestionario de 29 preguntas ${ }^{7}$, que se hizo público en la plataforma e-encuesta.com el 8 de febrero de 2016.

El cuestionario fue diseñado teniendo en cuenta todos los criterios que se utilizan habitualmente para la selección del tipo de preguntas a utilizarse, la elaboración de las mismas y su ordenación (Selltiz et al., 1980; Vroom, 1984; Hernández et al., 1997; Díaz, 2005; Trespalacios et al., 2005; Ramírez, 2007; Alvira, 2011;). Asimismo, se trató en todo momento de tomar como base los estudios de mercado existentes, por considerar que de ellos se podría extraer un conjunto de prácticas de utilidad ya constatada. En ese sentido, la gran mayoría de las preguntas se apegaron a lo realizado por otras investigaciones de mercado del TE. 
Hasta el 8 de junio de 2016, día en que se cerró la encuesta, fue necesario llevar a cabo una intensa tarea de promoción, la cual se desarrolló fundamentalmente a través de redes sociales, como Twitter y especialmente Facebook (aunque también se usaron otros medios, como comentarios en páginas web de medios de comunicación). La encuesta se dio así a conocer entre un público muy amplio, con 57,927 accesos totales al cuestionario ${ }^{8}$, que fue contestado por 1,362 de los que accedieron.

Una vez cerrada la encuesta en la plataforma, se procedió a depurar el informe proporcionado por e-encuesta.com, exportando en primer lugar los resultados a una hoja de cálculo y eliminando a grupos que no aplicaban (p.ej. de los menores de edad), así como quienes no contestaron datos demográficos clave como la edad o el sexo. El estudio quedó así con 1,205 encuestas válidas, cuyos principales hallazgos se expondrán a continuación.

\section{Resultados}

Los datos demográficos del estudio muestran una población con predominio del sexo femenino ( $55 \%$ frente a un $45 \%$ masculino) y en su mayoría joven (63\% entre 18 y 30 años, y $28 \%$ entre 31 y 45). Asimismo, predominan los solteros (51\%) y los casados (30\%) frente a las otras opciones de estado civil. Un 54\% no tienen hijos o dependientes económicos. En cuanto a su distribución territorial, el estado de México con mayor participación es Oaxaca, con 136 encuestados, lo cual hace un 11\% del total. Esta clara sobrerrepresentación de la población de dicho estado se debe al método de promoción, que se apoyó en un primer momento en las redes sociales personales. Después de Oaxaca, los estados con mayor participación son la Ciudad de México (99 encuestados), Aguascalientes (96), Chihuahua (95) y Puebla (60).

Un 75\% de los encuestados poseen nivel de Licenciatura; éste es un dato de interés, pues resulta consistente con los hábitos de consumo de Internet en México. En cuanto a los ingresos y el patrimonio, aspectos de gran importancia para el TS, sólo el 1\% de los encuestados gana más de 100,000 pesos mensuales, y sólo un 4\% posee un patrimonio de 10 millones de pesos o superior, a partir del cual se comenzaría a entrar en el segmento de quienes pueden realizar TS. Este es un dato interesante para la investigación, puesto que muestra la existencia de un grupo de encuestados que de manera realista podrían consumir TS.

Para estimar la demanda futura de TS en México, se trató de seguir un método semejante al de Tauri (2012), que tratase de establecer condiciones lo suficientemente restrictivas a los posibles demandantes como para seleccionar a personas que pueden y quieren comprar los productos ofrecidos. Así, lo primero que se hizo fue construir un indicador al que se denominó PC (prospecto cualificado). Este indicador estaba compuesto por personas que:

- Poseen un patrimonio igual o superior a 10,000,000 de pesos (dato obtenido en la pregunta número10).

- Estarían dispuestos a realizar turismo espacial suborbital "ahora mismo" (pregunta 23).

- Tienen un nivel de salud "bueno" o "excelente" (pregunta 24), y

- Han realizado alguna actividad de turismo de aventura (datos de la pregunta 12, excluyendo los cruceristas).

Haciendo los cálculos pertinentes, se halló que entre las 1,205 encuestas válidas que constituyeron el punto de partida de la investigación, existían 7 personas que cumplían estos cuatro requisitos, lo cual representa un $\mathrm{PC}=0.580912 \%$ de la población encuestada. Para corregir posibles efectos de insinceridad en las respuestas, se decidió tomar como base para el cálculo la mitad de la cifra obtenida, es decir, se tendría un PCfinal $=0.290456 \%$. Si esta cifra se aplicase al total de la población de México, que según la Encuesta Intercensal 2015 realizada por el Instituto Nacional de Estadística y Geografía (INEGI) ascendió a 119,530,753 habitantes (datos disponibles en la web del INEGI) nos arrojaría un total de 347,184 prospectos cualificados en México. Evidentemente, se trata de una cifra irreal, producto de los diversos sesgos ya señalados de la encuesta, como la juventud de los participantes y la sobrerrepresentación de personas con poder adquisitivo elevado (muy superior en proporción a la existente en la sociedad mexicana). Con respecto a este último elemento, la cifra obtenida es casi tres veces mayor que el número de millonarios existentes en México, que según la revista Forbes (2016) era en 2015 de 122 mil personas.

Teniendo en mente este problema, se optó por construir un indicador más restrictivo para que fuese aplicado al número de millonarios existentes, un dato que está disponible de manera confiable, al 
provenir de una fuente como Forbes, a diferencia de las consideraciones de salud o disposición a volar, que precisamente surgen de la encuesta realizada y cuyo establecimiento para el caso de la población total del país exigiría una investigación aparte. El segundo indicador PC' filtraría a las personas que:

- Estarían dispuestos a realizar turismo espacial suborbital "ahora mismo".

- Tienen un nivel de salud "excelente", y

- Han realizado alguna actividad de turismo de aventura.

Observando los datos de la encuesta, se encontró que 107 participantes en la encuesta cumplían con los tres requisitos mencionados, es decir $\mathrm{PC}^{\prime}=8.87 \%$. Para prevenir efectos de insinceridad, desconocimiento y también para contrarrestar el posible sesgo demográfico, con participantes en su mayoría jóvenes (los cuales por otros estudios se sabe que muestran una mayor disposición a realizar $\mathrm{TE}$ ), se decidió tomar como referencia únicamente la quinta parte de tal cifra, obteniendo así un $\mathrm{PC}_{\text {final }}=$ $1.774 \%$. Este valor fue aplicado entonces a los 122,000 millonarios existentes en México, obteniéndose una estimación inicial de 2,164 mexicanos que podrían ser prospectos para los vuelos suborbitales. De acuerdo con lo establecido por Tauri (2012: 31) se estimó que un 40\% de este total volaría durante los primeros diez años.

Desde luego, si todas las personas decidieran viajar en los primeros diez años a los que se refiere el pronóstico, las cifras de demanda serían mucho más altas. Sin embargo, se ha preferido, siguiendo el ejemplo de Tauri (2012), asumir una cifra conservadora. Se asumió que este $40 \%$ viajaría a un ritmo de $1 \%$ el primer año, creciendo después al 1.1, 1.3, 1.7, 2.5, 3.5, 4.7, 6.2 y 8\%, hasta llegar a un $10 \%$ en el último año, tratando de representar así la fase de introducción de un producto tan nuevo, hasta el inicio de la fase de crecimiento, según los modelos usuales del ciclo de vida del producto (véase Kotler et al., 2011: 323). Se trata, quiere recalcarse, de una forma muy conservadora de abordar el tema.

Asimismo, dado que el número de millonarios varía año con año, se trató de ponderar esa variación sumando a la cifra que se acaba de calcular un porcentaje, para lo cual se debió tomar en cuenta la variación del crecimiento en el número de millonarios en México. Según Forbes (2016), entre los años 2014 y 2015 se produjo un descenso en el crecimiento de tal grupo. Esta cifra, de acuerdo con Wealthinsight (2014) había sido precedida por un incremento muy fuerte en los años anteriores. Además, la firma Credit Suisse (2015: 43) previó entre 2016 y 2020 un incremento del 68\% en el número de millonarios, que alcanzará los 206,000 en la última fecha mencionada. Debido a todo ello, se asumió un incremento en el número de millonarios de $3 \%$ anual (lo cual se considera una cifra también muy conservadora) que se agregó así al cálculo.

Haciendo tales ajustes, la previsión de la demanda queda expresada en la siguiente tabla, que incluye también una previsión de los ingresos en pesos mexicanos generados por concepto de TS, tomando en cuenta un precio base de cuatro millones de pesos (equivalente aproximado a los 200,000 USD que se consideran como precio del TS, véase p. ej. Filgueiras, 2016).

Tabla 3: Escenario base. Previsión de la demanda de TS en México a diez años vista, con número de pasajeros e ingresos expresados en millones de pesos MXN.

\begin{tabular}{|c|c|c|c|c|c|c|c|c|c|c|}
\hline & 1er. año & $2^{\circ}$ & $3^{\circ}$. & $4^{\circ}$. & $5^{\circ}$. & $6^{\circ}$ & $7^{\circ}$ & $8^{\circ}$ & $9^{\circ}$ & $10^{\circ}$. \\
\hline Pax. & 9 & 10 & 12 & 16 & 24 & 35 & 49 & 66 & 88 & 113 \\
\hline Ingresos & 36 & 40 & 48 & 64 & 96 & 140 & 196 & 264 & 352 & 452 \\
\hline
\end{tabular}

Fuente: Elaboración propia.

Este será el escenario base. Se trata de cifras altamente conservadoras y que únicamente se refieren al segmento más adinerado de la población (sin tomar en cuenta los entusiastas del espacio de menor poder adquisitivo, que en otros estudios se considera podrían incrementar ligeramente la demanda). Aun así, se trata de cifras interesantes, sobre todo cuando se observa que el volumen de ingresos en estos diez primeros años de la previsión por los 422 turistas suborbitales mexicanos abarcaría un total de 1,688 millones de pesos (que equivaldrían a 84.4 millones de dólares, tomando el precio de 200,000 USD como constante, así como el tipo de cambio).

Se planteó también un escenario ampliado, en el cual se realizaron las siguientes modificaciones con respecto al escenario base. En primer lugar, se tomó un PC' con el valor de un cuarto de la cifra 
obtenida en la encuesta (es decir PC'ampliado=2.218\%), que representaría un total de 2,706 personas para el cálculo de los prospectos en el primer año. Asimismo, se consideró que un $60 \%$ de dichas personas volarían durante los primeros diez años, con tasas de 1.5\% el primer año, y posteriormente 1.9, 2.5, 3.3, 4.4, 5.7, 7.3, 9 y 10.9\%, hasta llegar al 13.5\% en el décimo año, es decir, el porcentaje que en los modelos habituales de tasa de adopción de nuevos productos se considera para los denominados adoptadores tempranos, véase Bigné et al., 2000). Por último, se consideró el incremento de la cifra de millonarios con un valor más cercano a la predicción de Credit Suisse, asumiendo un crecimiento del 5\% anual. Con estas modificaciones, la previsión quedaría como muestra la siguiente tabla:

Tabla 4: Escenario ampliado. Previsión de la demanda de TS en México a diez años vista, con número de pasajeros e ingresos expresados en millones de pesos MXN.

\begin{tabular}{|c|c|c|c|c|c|c|c|c|c|c|}
\hline & $\begin{array}{l}\text { 1er. } \\
\text { año }\end{array}$ & $2^{\circ}$ & $3^{o}$ & $4^{\circ}$ & $5^{\circ}$ & $6^{\circ}$ & $7^{\circ}$ & $8^{\circ}$ & $9^{\circ}$. & $10^{\circ}$. \\
\hline Pax. & 24 & 32 & 45 & 62 & 87 & 118 & 159 & 206 & 261 & 340 \\
\hline Ingresos & 96 & 128 & 180 & 248 & 348 & 472 & 636 & 824 & 1,044 & 1,360 \\
\hline
\end{tabular}

Fuente: Elaboración propia.

Con las nuevas condiciones establecidas, en este escenario ampliado, durante los diez años estudiados por la previsión, 1,334 mexicanos realizarían TS, generando (a un precio constante de cuatro millones de pesos) un volumen total de ingresos de 5,336 millones de pesos, equivalente a 266.8 millones de dólares.

Por último, se quiso plantear un escenario restringido, tomando en cuenta el mismo PC' del escenario base, es decir $\mathrm{PC}_{\text {restringido }}^{\prime}=1.774 \%$, lo cual arrojó una estimación de 2,164 prospectos, de los cuales se asumió que únicamente el $20 \%$ volaría durante los diez primeros años, con tasas de adopción de $0.3 \%, 0.5,0.8,1.1,1.5,2,2.5$, 3.1, 3.7 y un 4.5\% para el último año. Asimismo, se consideró un incremento anual del número de millonarios del 1.5\%, muy por debajo de lo previsto. En este escenario restringido, la previsión quedaría como sigue:

Tabla 5: Escenario restringido. Previsión de la demanda de TS en México a diez años vista, con número de pasajeros e ingresos expresados en millones de pesos MXN.

\begin{tabular}{|c|c|c|c|c|c|c|c|c|c|c|}
\hline & $\begin{array}{l}\text { 1er. } \\
\text { año }\end{array}$ & $2^{\circ}$ & $3^{\circ}$. & $4^{\circ}$. & $5^{\circ}$. & $6^{\circ}$. & $7^{\circ}$ & $8^{\circ}$. & $9^{\circ}$. & $10^{\circ}$ \\
\hline Pax. & 1 & 2 & 4 & 5 & 7 & 9 & 12 & 15 & 18 & 22 \\
\hline Ingresos & 4 & 8 & 16 & 20 & 28 & 36 & 48 & 60 & 72 & 88 \\
\hline
\end{tabular}

Fuente: Elaboración propia.

De acuerdo con este escenario restringido, se tiene que durante los diez años estudiados viajarán al espacio 95 mexicanos, generando unos ingresos de 380 millones de pesos. Sin duda, las cifras son inferiores a los escenarios previos, como cabría esperar, pero sigue tratándose de valores monetarios interesantes.

Con los resultados obtenidos en los tres escenarios, se construyó la siguiente tabla, para permitir una mejor comparación.

Tabla 6: Comparativo de los tres escenarios de previsión de la demanda de TS en México a diez años vista, según número de pasajeros.

\begin{tabular}{|c|c|c|c|c|c|c|c|c|c|c|}
\hline & $\begin{array}{l}\text { 1er. } \\
\text { año }\end{array}$ & $2^{\circ}$. & $3^{\circ}$. & $4^{\circ}$. & $5^{\circ}$. & $6^{\circ}$. & $7^{\circ}$ & $8^{\circ}$. & $9^{\circ}$. & $10^{\circ}$. \\
\hline Base & 9 & 10 & 12 & 16 & 24 & 35 & 49 & 66 & 88 & 113 \\
\hline Ampliado & 24 & 32 & 45 & 62 & 87 & 118 & 159 & 206 & 261 & 340 \\
\hline Restringido & 1 & 2 & 4 & 5 & 7 & 9 & 12 & 15 & 18 & 22 \\
\hline
\end{tabular}

Fuente: Elaboración propia. 


\section{Reflexiones finales}

La tabla que se acaba de exponer ofrece un buen punto de partida para comenzar a reflexionar sobre los hallazgos de la presente investigación. Dichos hallazgos parecen señalar que, hoy por hoy, en México podría existir ya una interesante demanda de clientes potenciales de TS. Para defender esta idea, se debe profundizar en primer lugar en las limitaciones del presente trabajo, las cuales son comunes a muchos estudios realizados a través de Internet. Se vuelve a hacer referencia así a algo que no ha dejado de recalcarse a lo largo de todo el estudio, como es el hecho de que el muestreo elegido, de tipo voluntario, no proporciona conclusiones extrapolables estadísticamente. Teniendo en cuenta esta grave limitación, se establecieron una serie de condiciones encaminadas a darle a los escenarios el mayor realismo posible, en especial al seleccionar a las personas que pueden y quieren hacer TS, para aplicarla después al grupo de personas del cual, según la práctica totalidad de los estudios consultados, provendrán los primeros turistas suborbitales.

Es obvio que siempre existe un elemento de incertidumbre común a todos los estudios de mercado sobre el TE, pues existen muchos imponderables que podrían afectar las previsiones. Se trata de aspectos como el crecimiento o contracción de la economía, la intensidad y efectividad de las campañas de mercadotecnia, etc. Un elemento de enorme importancia son las fluctuaciones en el precio, dado que cuánto más descienda éste, más crecerá la demanda. Sin embargo, se decidió no tomar en cuenta tal factor en la construcción de los escenarios, debido sobre todo al ejemplo del estudio de Tauri (2012), que optó en todo momento por enfocar el tema desde una perspectiva restringida, sin pecar de exceso de optimismo, y que por ello asumió un precio constante, igual al manejado actualmente. El único aspecto que se añadió al estudio fue la ponderación del crecimiento anual en el número de millonarios, que se considera modifica sustancialmente la base sobre la que establecer los cálculos, sin hacerlos perder realismo, al ser una cifra proveniente de fuentes confiables.

Cuando se analizan en sí mismos los datos de la encuesta, saltan a la vista los sesgos del misma. Sin embargo, analizar tales sesgos también aporta pistas que podrían ser relevantes de cara a momentos en los que el TS constituya una industria mucho más desarrollada. En ese sentido, aunque puede afirmarse sin ninguna duda que la gran mayoría de los participantes en la encuesta no tienen el nivel económico requerido para consumir productos de TS al precio actual, lo cual es un dato negativo. Pero los datos de interés por el TS y la disposición a realizarse parecen ser positivos: en un plazo de tiempo más amplio, y suponiendo una paulatina reducción de los precios, la cifra de interés manifestada por los encuestados, con un 67\% de personas que realizarían "ahora mismo" TS de disponer del dinero necesario, comenzará a cobrar cada vez mayor importancia.

Desde luego, se tiene plena conciencia de que el TE (en cualquiera de sus modalidades) es un tema muy complejo, y que obligadamente se han tenido que dejar fuera de la investigación numerosos aspectos de gran interés. Un ejemplo de estos factores ha sido el precio, como se acaba de comentar, cuya influencia en la demanda suele ser directa y decisiva, según muestra la teoría económica. Pero no es el único: los aspectos político-legales, pueden tener también una enorme influencia sobre el desarrollo futuro de la industria espacial (véase p.ej. la recopilación de Wouters et al., 2017). Al respecto, dado que en México no existen actualmente empresas oferentes de TE (las pocas agencias que ofrecen esta modalidad turística se limitan a comercializar productos ofrecidos por compañías extranjeras), se deberá estar pendiente de lo que suceda en Estados Unidos, el lugar desde el que previsiblemente volarán los primeros turistas suborbitales. Las regulaciones en torno a la seguridad son particularmente importantes, dado que pueden llegar a paralizar los vuelos.

Aun así, se considera que el valor de un estudio como el presente debe ponderarse desde la perspectiva expresada por Collins et al. (1994), la cual mantiene su vigencia en la actualidad: la industria del TS se encuentra aún en los momentos iniciales de su desarrollo, y éste no puede detenerse hasta que exista información que garantice una plena certeza. Esta perspectiva legitima el uso de los datos disponibles, a pesar de sus limitaciones.

Desde luego, ello no quiere decir que se deban obviar tales limitaciones; más bien, se trata de exponerlas de modo claro, de modo que aquellos para quienes los datos puedan resultar de interés puedan evaluar su utilidad como guía en la toma de decisiones. Se considera que éste es el modo adecuado de entender los resultados de la presente investigación, que muestra con cierta plausibilidad la existencia de una demanda potencial para los primeros años del TE.

\section{Agradecimientos}

A Sofía Lorena Rodiles Hernández, Mayra Manuel Aragón, Emmanuel Arrazola Ovando, Jorge Alfonso Ramírez Luna y José Gastón García Flores, por sus valiosas revisiones y sugerencias. Se agradece también a un dictaminador anónimo de la revista PASOS su señalamiento de corregir el "doble filtrado" de millonarios, lo cual provocó interesantes cambios con respecto a los resultados iniciales. 


\section{Bibliografia}

Alvira Martín, Francisco

2011. La encuesta: una perspectiva general metodológica. Madrid: Centro de Investigaciones Sociológicas. Anderson, Eric y Piven, Joshua

2005. Space Tourist's Handbook. Where to go, what to see, and how to prepare for the ride of your life.

Philadelphia: Quirk Books.

Anónimo

2012. “Cuánto es un buen CTR en Google Adwords?”. Spanish SEO blog, febrero [http://spanishseoblog.

blogspot.mx/2012/02/cuanto-es-un-buen-ctr-en-google-adwords.html, consultado el 4 de julio de 2016] Beard, Suzette y Starzyk, Janyce

2002. Space Tourism Market Study. Orbital space travel \& destinations with suborbital space travel.

Bethesda, MD: Futron Corporation.

Bigné, J. Enrique, Font, Xavier y Andreu, Luisa

2000. Marketing de destinos turísticos: análisis y estrategias de desarrollo. Madrid: ESIC Editorial.

Burns, Alvin C. y Bush, Ronald F.

2003. Marketing research: online research applications. Nueva Jersey: Prentice Hall.

Collins, Patrick, Iwasaki, Yoichi, Kanayama, Hideki y Ohnuki, Misuzu

1994. "Commercial Implications of Market Research on Space Tourism". Journal of Space Technology

and Science: Special Issue on Space Tourism, parte 2, 10(2): 3-11. [http://www.spacefuture.com/ archive/commercial_implications_of_market_research_on_space_tourism.shtml, consultado el 5 de agosto de 2009]

Collins, Patrick, Stockmans, Richard y Maita, M.

1995."Demand of space tourism in America and Japan, and its implications for future space activities".

AAS (American Astronautical Society), paper AAS no. 95-605, vol. 91: 601-610.

Credit Suisse

2015. Global Wealth Report 2015. Zürich: Credit Suisse Research Institute.

Díaz de Rada, Vidal

2005. Manual de trabajo de campo en la encuesta. Madrid: Centro de Investigaciones Sociológicas.

Dobson, Jim

2015. "Virgin Galactic Tells Billionaire Passengers, SpaceShipTwo Launch in 2017", Forbes, 28 de Julio [http://www.forbes.com/sites/jimdobson/2015/07/28/virgin-galactic-spaceshiptwo-tells-billionaire-passengers-fall-2017-is-new-launch-date/\#502ced7840c2, consultada el 6 de Agosto de 2015]

Estaella, Adolfo y Ardévol, Elisenda

2011. "E-research: desafíos y oportunidades para las ciencias sociales", Convergencia, 55: 87-111.

Excelsior

2014. "Se estrella nave espacial de Virgin Galactic en el desierto de Mojave", Excelsior, 31 de octubre [http://www.excelsior.com.mx/global/2014/10/31/989923, consultada el 19 de julio de 2015].

Filgueiras-Nodar, José María

2015. "Turismo Espacial", seminario impartido en la Universidad del Mar (campus Puerto Ángel), Oaxaca, México, el día 28 de abril.

2016. "Los precios en el turismo espacial", Turismo espacial en español, junio [https://turismoespacial. com.mx/2016/06/01/los-precios-en-el-turismo-espacial/, consultada el 3 de enero de 2017]

Forbes

2016. "Hay 122,000 millonarios en México; 50,000 menos que en 2014", Forbes, 4 de julio [http://www. forbes.com.mx/hay-122000-millonarios-en-mexico-50000-menos-que-en-2014/, consultado el 4 de julio de 2016]

Ghoshal, Mandira

2007. "Space Tourism: Dream or Reality?". En: Harish, R (ed), Space Travel and Tourism. New Frontiers (pp. 54-71). Hyderabad, India: The Icfai University Press.

Hernández Sampieri, Roberto, Fernández Collado, Carlos y Baptista Lucio, Pilar

1997. Metodología de la investigación. México: McGraw Hill.

Jakhu, Ram S.

2010. Space tourism in India. Dehradun, India: University of Petroleum and Energy Studies.

Jiménez, Carlos y Puente, Raquel

2007. "La investigación de mercados online: ¿realmente funciona?". Debates Iesa, 12(2): 20-24. 
Kotler, Philip, Bowen, John T., Makens, James C., García de Madariaga, Jesús y Flores Zamora, Javier 2011. Marketing turístico. Madrid: Pearson.

La Jornada

2013. "Hay en México 145 mil millonarios y 2,540 multimillonarios: informe", La Jornada, 19 de junio. López Romo, Heriberto

2009. "Los niveles socioeconómicos y la distribución del gasto" [http://www.amai.org/NSE/NivelSocioeconomicoAMAI.pdf, consultado el 28 de agosto de 2015]

Morales Vargas, Gonzalo

2011. La investigación de mercados online y la netnografía. Seminario para optar al título de Ingeniero

Comercial, Facultad de Economía y Negocios, Universidad de Chile, Santiago.

Piñón-González, María Angélica

2016. El mercado del turismo espacial suborbital en México: estudio a través de Internet del comportamiento del consumidor potencial y pronóstico de la demanda futura. Tesis de Licenciatura no publicada, Universidad del Mar, Bahías de Huatulco (México), 12 de septiembre.

Ramírez de la Roche, O.F.

2007. "Obstáculos para la elaboración de escalas tipo Likert: una reflexión personal". Archivos en Medicina Familiar, 9(2): 71-74.

Randolph, Joanne

2003. Dennis Tito. First Space Tourist. Nueva York: Rosen.

Sadeh, Eligar y Sadeh, Cris

2011. "X Prize Foundation”. En: Sadeh, Eligar (ed.), The Politics of Space: a Survey (p. 306). Londres y Nueva York: Routledge.

Seedhouse, Erik

2008. Tourists in Space. A practical guide. UK: Springer-Praxis.

Seedhouse, Erik

2014. Suborbital. Industry at the edge of space. Suiza: Springer-Praxis.

Seedhouse, Erik

2015. Virgin Galactic: the first ten years. Chichester, UK: Springer-Praxis.

Seedhouse, Erik

2016. XCOR, Developing the next generation spaceplane. Suiza: Springer-Praxis.

Selltiz, Claire, Jaahoda, Marie, Deutsch, Morton y Cook, Stuart W.

1980. Métodos de investigación en las relaciones sociales. Madrid: Rialp.

Tauri- The Tauri Group LLC.

2012. Suborbital Reusable Vehicles: A 10 Years Forecast of Market Demand [http://www.nss.org/transportation/Suborbital_Reusable_Vehicles_A_10_Year_Forecast_of_Market_Demand.pdf, consultada el 22 de Agosto de 2015]

The Guardian

2014. "Some Virgin Galactic seatholders ask for refund on tickets after crash", The Guardian, 5 de noviembre [http://www.theguardian.com/science/2014/nov/05/virgin-galactic-seatholders-ask-for-refund-on-tickets, consultada el 22 de Julio de 2015]

Trespalacios Gutiérrez, Juan Antonio, Vázquez Casielles, Rodolfo y Bello Acebrón, Laurentino

2005. Investigación de mercados. Métodos de recogida y análisis de la información para la toma de decisiones en marketing. Madrid: Paraninfo.

Umashankar, Roopa

2007."Virgin Galactic: Game plans for space tourism". En: Harish, R. (ed), Space Travel and Tourism. New Frontiers (pp. 186-197). Hyderabad, India: The Icfai University Press.

Vroom, Victor H.

1984. Work and motivation. Londres: National Book Network.

Wealthinsight

2014. "The 2014 millionaire explosion", [http://www.wealthinsight.com/pressrelease/the-2014-millionaire-explosio-4161554, consultada el 19 de agosto de 2015]

Webber, Derek y Reifert, Jane

2006. "Filling in some gaps". Executive summary of The Adventurer's Survey of Public Space Travel. Bethesda, MD: Spaceport Associates.

Wouters, Jan, De Man, Philip, Hansen, Rik (eds)

2017. Commercial uses of space and space tourism. Cheltenham, UK y Northampton, USA: Edward Elgar. 
Zikmund, William G. y Babin, Barry J.

2009. Investigación de mercados. México: CENGAGE Learning.

\section{Notas}

1 De dichos clientes, se estima que tan sólo 20 han cancelado sus reservaciones después del accidente (The Guardian, 2014), lo cual muestra la confianza de que goza Virgin entre su público

2 Evidentemente, la precisión está limitada en gran medida por el carácter novedoso del TS. Sin duda, los pronósticos serán mucho más acertados después del inicio de los vuelos.

3 De éstos, 2,272 poseían un patrimonio superior a los 2 mdd., 252 superior a los cien mdd., y 16 superior a los mil mdd.

4 Éste, obviamente, posee una alta sensibilidad al tiempo, ya que, como explica Tauri (2012), si por ejemplo todos los individuos estimados volasen en la primera década, la demanda se duplicaría.

$5 \quad$ En Hernández et al. (1997: 213) se afirma que la elección de una muestra probabilística o no probabilística depende de varios elementos, como el diseño de la investigación y la contribución que se planea aportar con la misma. Atender a estos elementos ayuda a justificar la elección del muestreo, que resulta adecuado para un diseño no experimental. Lo mismo ocurre con la contribución: al igual que ha sucedido con varios estudios de mercado del TE mencionados (Webber y Reinfert, 2006; Ghoshal, 2007), la investigación pretende proporcionar cifras tentativas capaces únicamente de orientar posibles decisiones comerciales, lo cual puede hacerse a través de un muestreo no probabilístico.

6 Evidentemente, la principal desventaja de la encuesta es que no permite la profundización en las respuestas, al ofrecer únicamente la elección entre opciones preestablecidas. Para dejar abierta la posibilidad de que los encuestados ofrecieran respuestas más personales, se incluyó un apartado de comentarios adicionales, que fue contestado por numerosos participantes y que está siendo objeto de un análisis cualitativo.

7 Las 29 preguntas exploraban otros aspectos, como por ejemplo las actitudes del público ante el TE, su percepción general del mismo, sus consideraciones acerca de los precios que se considerarían justos o las razones que los motivarían a comprar tal producto turístico (véase Piñón, 2016). Para la investigación expuesta en el presente artículo se utilizaron únicamente cuatro de dichas preguntas, además de los datos demográficos que se señalarán.

8 En referencia a la publicidad online de Google, se comenta que "un CTR [click-through-rate] medio es, probablemente, cercano al 2\%" (Anónimo, 2012). Tomando en consideración este CTR, los enlaces y anuncios promocionales que dirigían hacia la encuesta habrían sido vistos aproximadamente por 2,896,350 personas. Dado que los anuncios de Google AdWords poseen una gran personalización y se enfocan por ello en grupos a los que en principio pueden interesar, es probable que el número de personas alcanzadas por la promoción del cuestionario haya sido mucho mayor. 